\title{
文物建筑迁移保护中的场地利用与环境塑造
}

森凡

天津大学建筑设计研究院

DOI:10.18686/bd.v1i12.1141

[摘 要] 文物建筑是能够反映历史风貌、地方特色, 且具有一定文物价值的建筑。在我国社会经济快速发展的背景下, 文物 建筑保护工作变得越来越重要, 要求也越来越高。基于此, 本文就对文物建筑迁移保护中的场地利用与环境塑造展开探究, 以 期为文物建筑迁移保护工作提供参考依据。

[关键词]文物建筑; 迁移保护工作; 场地利用; 环境塑造

文物建筑迁移保护工作展开的原因是一些历史文物建 筑无法在当地展开保护保护工作, 所以必须将这部分文物 建筑迁移到其他地方进行保护, 避免文物建筑出现损坏等 情况。基于此, 本文就对文物建筑迁移保护的概念进行阐述, 并探讨文物建筑迁移保护中的场地利用与环境塑造的主要 对策,以期提高文物建筑迁移保护工作质量。

\section{1 文物建筑迁移保护的概念分析}

一般情况下, 我国文物建筑保护包含原地保护和迁移 保护两种, 顾名思义, 文物建筑迁移保护就是指, 将文物建筑 移送到其他地点进行保护, 是我国文物建筑保护中一种常 使用的方法。文物建筑宿迁保护主要是针对城市中的一些 大型建筑,这些建筑由于一些原因,在当地无法正常进行保 护,所以必须移送到指定地区进行保护, 实现文物建筑保护 工作目标, 文物建筑迁移保护主要包含文物建筑局部迁移 和文物建筑整体迁移这两种,例如,我国在建设安林高速公 路时, 为了保证周围的慈源寺不受影响, 就对慈源寺进行了 整体迁移工作; 而重庆市在建设三峡库时, 为了保障奉节县 的㫿塘峡壁摩崖题刻不受破坏, 就展开了局部迁移工作,对 这些文物建筑进行了保护。需要注意的是,相关单位在展开 文物建筑迁移保护工作时, 需要从文物建筑整体出发, 并对 文物建筑迁移地点进行研究, 保证文物建筑和文物迁移地
点可以完美融合在一起, 保证文物建筑迁移到指定地点后, 既不会丧失文物建筑的历史价值, 也不会对迁移地周围环 境产生影响,从而保证文物迁移保护工作质量。

\section{2 文物建筑迁移保护中的场地利用分析}

2.1 文物建筑对未开发新址中自然山水地形的利用分 析

文物建筑在迁移过程中所选择的迁移地点具有多种类 型, 如果文物建筑迁移到未开发新址时, 需要加强对新址中 的自然山水地形的利用,具体可以从以下两个方面展开:一 方面, 相关人员在进行迁移地质选择过程中,需要对迁移地 质的地形条件、可利用空间、生态环境情况等方面内容进行 分析, 保证所选择的迁移地点与文物原先所处环境相似, 迁 移场地的面积、方位都与文物建筑相符合。另一方面,相关 人员确定了文物迁移地质后, 还需要对迁移地质周围的地 形、土壤、植被、水体等自然环境进行了解, 根据迁移地质周 围的自然环境选择迁移文物,让文物建筑做到“因地制宜”, 能够与开发新址中自然山水地形完美融合在一起, 实现文 物建筑迁移保护目标。例如,相关人员在对湖北省剓归县的 文物建筑进行迁移保护工作时, 所选择的文物建筑迁移新 址在风凰山景区, 面积大约为 8.20 平方千米, 相关人员对迁 移新址进行分析后, 对迁移新址重新展开布局,根据不同地 
形条件选择不同的文物建筑,然后进行文物建筑迁移,让文 物建筑和周围环境完美的融合在一起, 实现了文物建筑对 未开发新址中自然山水地形的有效利用。在重庆市巫县大 昌民居迁移工作时, 相关人员所选择的迁移地点为主镇区 东部西包岭地段,这个地段位于大宁河的北岸,与文物建筑 原厂地有着相似之处, 文物建筑迁移后可以更好进行融合, 实现文物建筑保护工作目标。

2.2 文物建筑对已有建成环境中人工场地的利用分析

相关单位在文物建筑迁移保护工作中经常会将迁移保 护地点选择在已有建成环境中人工场地, 在此种情况下, 就 需要注意以下两个方面内容:一方面, 相关人员需要根据已 有建成环境中人工场地的大小、环境特点等内容合理选择 建筑物,保证建筑物色彩、样式与场地能够完美融合, 提高文 物建筑宿迁保护质量。另一方面,由于部分文物建筑群具有 一定特征,在此种情况下,相关人员就需要保证文物建筑迁 移场地能够与建筑群的气氛相协调, 保证文物建筑迁移工 作对场地能够合理利用, 为已有建成环境中人工场地营造 一个人文景观。例如,在广西壮族自治区北海市英国领事馆 迁移时, 相关人员所选择的迁移场地为广西北海市的一个 校园内部, 这个新场地周围有法国天主堂北海教区主教府 楼、教会医院、德国领事馆旧址等建筑,这些建筑都具有西 式风格,将北海市英国领事馆迁移到这个场地,可以与周围 文物建筑完美融合, 从而形成一个独特的人文景观, 增加文 物建筑价值。

\section{3 文物建筑迁移保护中的环境塑造分析}

3.1 文物建筑迁移保护的空间特色塑造工作分析

相关人员要想保证文物建筑宿迁保护工作质量, 就需 要加强迁移地质的空间特色塑造工作, 具体可以从以下两 个方面展开:一方面,相关人员需要保证文物建筑本身特点 不被掩埋。在展开文物建筑宿迁保护工作时, 相关人员需要 加强新场地的选择工作, 让文物建筑可以与新场地周围环 境完美融合, 不受限制。另一方面,相关人员在展开空间塑造 工作时, 需要将一些空间不合格的建筑物进行拆除工作, 避 免文物建筑发生重复。例如,相关人员在对张桓侯庙建筑进 行迁移时,由于所选择的地质岩层比较浅, 在此种情况下,相 关人员要想保证张桓侯庙建筑与周围环境完美融合, 就需 要使用钢筋混凝土对新场地进行浇筑工作, 形成一个屋基 后在展开文物建筑迁移工作,与此同时,相关人员还需要使 用人造岩石对新场地进行修饰工作，让新场地在修建过程 中不会出现问题,保证文物建筑迁移工作质量。与此同时,相 关人员在对河南省洛阳市新安县西沃乡西沃村的西沃石窟 进行迁移保护时, 由于此文物建筑具有自己的特点,规模也 偏大,在此种情况下,相关人员在展开文物迁移工作时, 就提 前对文物建筑制定了七根水泥挑梁，使用这七根水泥挑梁 为文物建筑搭建栈道,并使用毛石对文物建筑的将背部、顶 部和左右两侧包砌,将新砌的正面部分作旧,使新老岩石的 色调基本协调,让各个块体形成一个整体,能够与新场地完
美融合在一起,展现出文物建筑的具体特色。

3.2 文物建筑迁移保护的人文环境塑造工作分析

在文物建筑迁移保护工作中, 相关人员要想保证文物 迁移工作质量, 就需要加强周围人文环境塑造工作,具体可 以从以下两个方面展开:一方面, 文物建筑对人们有着吸引, 可以体现出历史发展特色, 在此种情况下, 相关人员就需要 严格遵照历史要求, 对历史文化元素进行考虑,让文物建筑 可以对迁移地的人文环境进行塑造, 让前去游玩的人们产 生思想共鸣。另一方面,相关人员需要保证文物建筑迁移工 作不会抹杀文物价值, 让文物建筑和人们文化活动进行融 合, 提升文化建筑的生命力, 实现人文环境塑造工作。例如, 相关人员在展开张桓侯庙迁移工作时, 为了保证文物建筑 的特色能够传承下去, 就需要选择随县城迁移的方法, 让张 桓侯庙民间祠庙的特点继续延续下去, 为当地居民定去举 办富有民俗色彩的活动, 让张桓侯庙的历史文化特色得到 传承, 从而为迁移地点营造一个良好的人文环境, 实现文物 建筑迁移保护工作目标。与此同时,相关人员在重庆市丰都 秦家大院的迁移工作中将文物与旅游行业相结合, 在文物 建筑中增加了人们活动区域, 让人们在文物建筑内可以进 行观赏、活动,从而打造一个集“可居性”、“可观性”、“可游 性”于一体场所,让文物建筑的原有价值得到传承, 更好的与 迁移环境相融合, 实现文物迁移工作的初衷。

\section{4 总结语}

总而言之, 文物建筑迁移保护工作是建筑保护的一种 方法,由于此项工作涉及地理学、环境学、建筑学、生物学等 诸多学科, 所以具有复杂性特点,在迁移保护工作中需要注 意的事项也非常多。面对此种情况,相关单位要想保证文物 建筑迁移保护工作质量, 就需要加强文物建筑与迁移地点 的研究工作, 保证文物建筑与迁移地点周围环境能够完美 融合, 降低对周围环境的影响, 实现文物建筑迁移保护工作 目标。

参考文献:

[1]原显涵,张晓巍.历史保护建筑与老城更新- - 以新 疆奇台县会馆片区为例 [C].2014 中国城市规划年会论文 集.2014:1-10.

[2]赵映清. 文物建筑保护与利用分析[J]. 文化学刊, 2017,(10):114-115.

[3]朱亚红,杨毅哲.城市化背景下古建筑的保护与利用 问题探析[J]. 建材发展导向,2017,15(04):32-34.

[4]林黎笛.浅谈文物建筑(群)保护的一些问题[J].福建 建设科技,2016,(03):39-42.

[5]王宇, 王力霞.历史城市与文物建筑保护研究 [J]. 建 设科技,2016,(24):63-64.

[6]胡彦羽.古建筑保护研究情况述评 [J]. 古建园林技 术,2016,(01):64-67.

[7]肖东.文物建筑搬迁保护中的场地利用与环境塑造 [J].古建园林技术,2017,(04):47-48+62. 